\title{
Review Article \\ The Effects of Very-Low-Calorie Diets on HDL: A Review
}

\author{
Catherine Rolland and Iain Broom \\ Centre for Obesity Research and Epidemiology, Robert Gordon University, St. Andrew Street, Aberdeen AB24 3LR, UK \\ Correspondence should be addressed to Catherine Rolland, c.rolland@rgu.ac.uk
}

Received 30 August 2010; Accepted 25 November 2010

Academic Editor: Andrew Brown

Copyright (C) 2011 C. Rolland and I. Broom. This is an open access article distributed under the Creative Commons Attribution License, which permits unrestricted use, distribution, and reproduction in any medium, provided the original work is properly cited.

This paper investigates the effects of very-low-calorie diets (VLCDs) used in the treatment of obesity on high-density lipoprotein (HDL) levels. Although the studies varied widely in their intervention format, duration, and baseline HDL levels, it would appear that HDL levels usually decrease during active weight loss using a VLCD, but these either return to pre-VLCD levels or improve overall during the weight-maintenance phase. More research needs to be done to determine optimal weight-maintenance programmes and the effects of VLCDs in the short term as well as on HDL levels in groups at increased risk of coronary heart disease.

\section{Introduction}

With the continuous rise in obesity, there has been resurgence in the use of very low calorie diets (VLCDs) and more research investigating the effects of such diets has been undertaken. VLCDs have often been criticised for being unsafe and an unhealthy way to lose weight. The current VLCDs, however, are not to be confused with those from the 1970s which resulted in a number of deaths, probably due to vitamin and mineral deficiencies and poor quality or inadequate amounts of protein $[1,2]$. VLCDs are defined as diet replacements with $<800 \mathrm{kcal}$ and $>400$ to $450 \mathrm{kcal} / \mathrm{d} \mathrm{[3]}$. There are now a number of commercially available VLCDs.

Evidence from a number of reviews [3-5] clearly demonstrates that VLCDs can result in significant weight loss. Despite this, there are still concerns about weight regain following these diets as well as detrimental health effects due to the rapid weight loss they induce. In this paper, we aim to examine the literature and to investigate the effects of very low calorie diets on high density lipoproteins (HDL) levels specifically.

\section{Importance of HDL}

Obesity is commonly associated with elevated triacylglycerols, low HDL, and normal low-density lipoproteins [6].
The low HDL observed in obesity is associated with an enhanced risk of atherogenesis possibly due to increased degradation and/or decreased production of HDL particles. Adipose cells have been shown to bind to HDL [7], hence increased body fat may lead to an increased uptake of HDL particles from circulation resulting in a reduction in plasma HDL levels [8]. On the other hand, an increase in HDL has been associated with decreased risk of coronary artery disease [9], probably due to its role in the reverse cholesterol transport process where cholesterol in peripheral tissues is transported to the liver for reuse or bile acid synthesis, preventing the accumulation of cholesterol in the arteries [10]. HDL is also thought to be cardioprotective due to its antioxidant activity [11].

\section{Effect of VLCD on HDL Levels}

Several studies have investigated changes in HDL after a period of VLCD. In Table 1, we report the findings from 5 studies in which participants underwent VLCDs for as little as 2 days and as long as 12 weeks. The calorie intake varied from 450-800 kcal/day. One study [12] looked at the differences in ethnicity in response to a VLCD delivered with exercise and behavioural therapy. Also, the gender composition of the studies were quite different, as some studies looked at women only $[12,13]$, while the other 
studies all included men [14-16]. Weight loss was significant in all but the 2-day VLCD group [13].

With the exception of the group that underwent a VLCD for 2 days [13], changes in HDL are reported in Table 1. HDL was significantly decreased in two of the studies $[12,14]$. Hong et al. [12] investigated the differences in response between black and white individuals. Their findings demonstrated that there was no difference between these two ethnic groups for weight and HDL in response to the VLCD intervention (which also included behaviour therapy and exercise).

Two other studies also displayed a trend for a decrease in HDL $[13,16]$. In the study by Lin et al. [16], all patients underwent two weeks of an LCD followed by 12 weeks of VLCD. The two groups differed in the caloric intake during the VLCD period (450 versus $800 \mathrm{kcal} /$ day). Weight loss and changes in HDL were not significant between the two groups.

Only one study reported an increase in HDL after a 9week VLCD [15], but this was not significant.

\section{Effect of VLCD and a Weight-Maintenance Period on HDL Levels}

A number of studies investigated changes in HDL after a VLCD and a weight-maintenance period (Table 2). These studies varied in duration of VLCD (6 weeks-9 months), maintenance period/followup (4 weeks-5 years). Energy intake for the VLCD varied from $400-800 \mathrm{kcal} / \mathrm{d}$. Two studies included exercise $[17,18]$, while three other studies included behaviour therapy [19-21], four studies included medication [22-25] and two studies investigated individuals with type 2 diabetes $[21,26]$. Weight loss was significant for all studies after the VLCD, although one study [18] included an LCD phase before the VLCD, another study [21] included a refeeding phase before the measurements were taken, and a third study reported BMI but not weight, and BMI change was not reported after the period of VLCD [26]. Weight loss at the end of the study remained significant in 9 of the 12 studies. As mentioned previously, the study by Paisey et al. [26] only reported BMI which did not change significantly. The changes in the study by Fogelholm et al. [18] were more difficult to report for both weight and HDL as the baseline values were reported for all of the participants and not provided separately for each treatment group. In addition, this study did not give within group significance for changes in weight or HDL at followup. Finally, Apfelbaum et al. [25] only report weight change from the time of randomisation after the VLCD to the end of the study, therefore not including the weight loss achieved using the VLCD.

At the end of the VLCD phase, HDL was reported for 9 of the 12 studies. HDL was decreased in at least one of the treatments for 6 of the studies [18, 20, 22, 23, 27, 28]. This was significant in three studies $[20,22,28]$ and not reported in two others [18, 23]. Four studies [19, 21, 24, 27] reported an increase in HDL after the VLCD, only one of which was not significant [27], however, the 2 studies that report an increase in HDL had a period of refeeding before the measurement $[19,21]$. All of the studies reported an increase in HDL at the end of the study with the exception of Haugaard et al. [28] and one arm in the study by Christiansen et al. [17] which both showed no change in HDL. The increase in HDL was significant in at least one arm of $8[17,19-22,24,25,27]$ out of the 12 studies.

The study by Christiansen et al. [17] reports that exercise results in a significant improvement on HDL while the study by Fogelholm et al. [18] shows a similar trend, but the results were not significant at the 2 year followup.

Delbridge et al. [27], do not report a significant difference in the improvement in HDL at the time of followup between the high-carbohydrate and the high-protein weightmaintenance group. Also, there were no significant differences in HDL between the use of a placebo and Orlistat [22] or between placebo and the neuropeptide 5 receptor antagonist MK-0557 [23] for weight maintenance, but there was a significant improvement in HDL when Sibutramine was used compared to placebo [25].

\section{Effect of Intermittent Use of VLCD on HDL Levels}

Five studies reported the effects of intermittent use of VLCDs on HDL (Table 3 ). The duration of the studies varied from 14 weeks to over two years. The energy intake from VLCDs ranged from 400-600 kcal/d. Two studies included women only [29] and one study included type 2 diabetic patients only [30]. The format of the different interventions varied widely, and is described in more detail in Table 3.

Only three studies reported weight loss after the first phase of VLCD $[8,21,31]$ and all three studies report a significant weight loss. Weight loss at the end of the study was significant for all five studies.

HDL change was reported after the first phase of VLCD in all 5 studies. At this stage, HDL had decreased significantly in 4 studies $[8,29,30]$, and increased in one study [21]. At the end of the studies, HDL was increased in at least one arm of 3 studies $[21,30,31]$, only two of which were significant [21, $31]$. HDL decreased in at least one arm of 3 studies $[8,29,30]$ none of which were significant.

\section{Discussion}

Here we reviewed the limited information available about the effects of VLCDs available. The results suggest that although an improvement in HDL levels during active weight loss using a VLCD is not always present, there often is an improvement observed during weight maintenance.

It was interesting to see in the study by Lin et al. [16] that there was no significant difference in weight loss and HDL between the group that consumed $450 \mathrm{kcal} / \mathrm{d}$ compared to the group that consumed $800 \mathrm{kcal} / \mathrm{d}$. This is consistent with other studies that compared the use of diets ranging from $240-880 \mathrm{kcal} / \mathrm{d}$ and found no significant difference in weight loss for short (4-6weeks) and intermediate term (1626 weeks) [32-34]. In light of this, it could be beneficial for patients to be allowed to consume more and minimise the sense of limitation that occurs when using a VLCD, and use a 


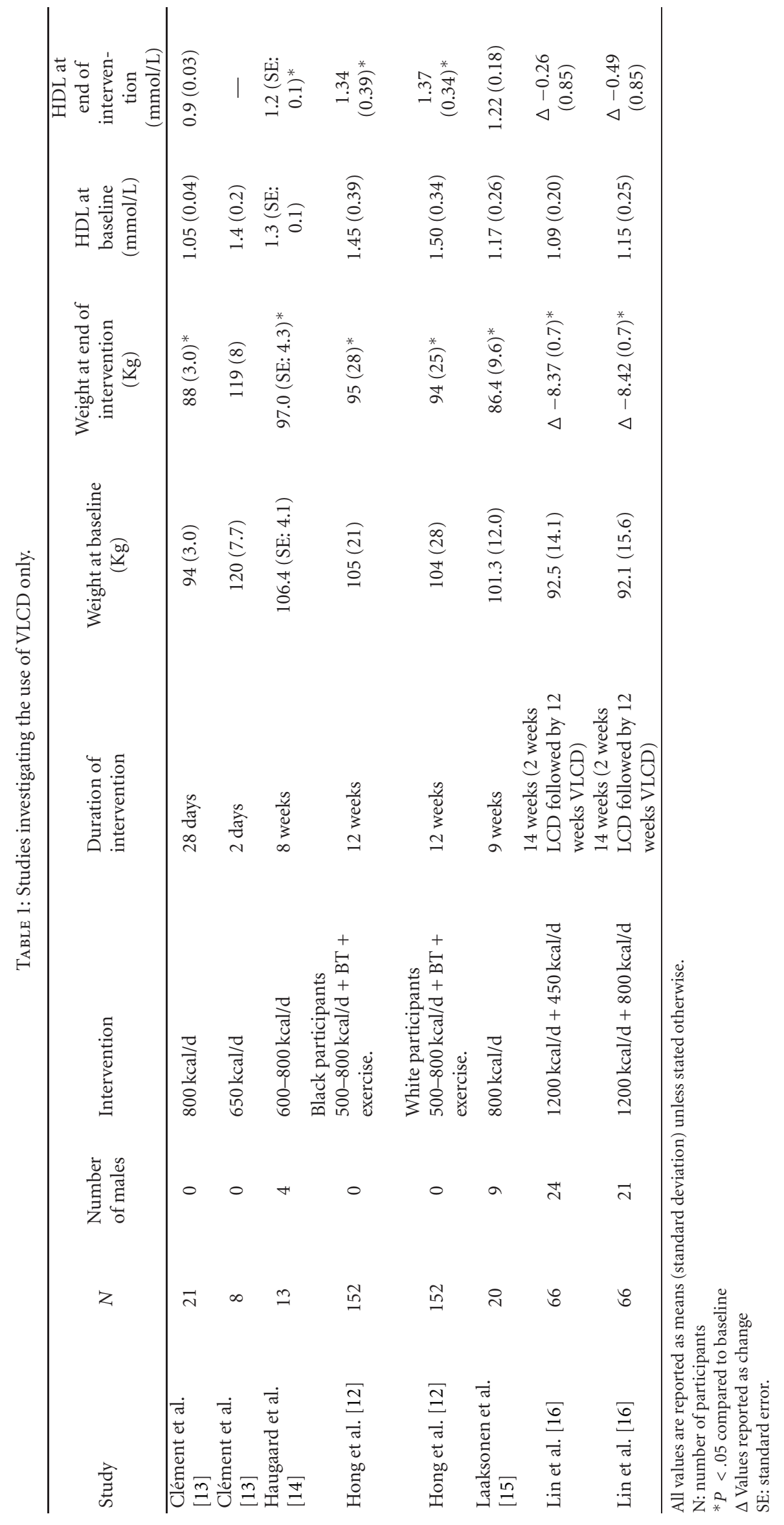




\begin{tabular}{|c|c|c|c|c|c|c|c|c|c|c|c|}
\hline 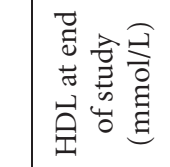 & $\begin{array}{l}0 \\
0 \\
0 \\
0 \\
0 \\
0 \\
0 \\
4\end{array}$ & $\stackrel{3}{\bullet} \stackrel{*}{\circ}$ & 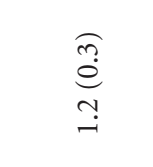 & $\begin{array}{c}\stackrel{*}{o n} \\
\stackrel{n}{\infty} \\
\stackrel{n}{-}\end{array}$ & 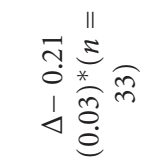 & 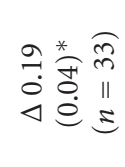 & 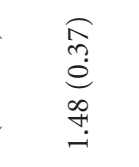 & $\begin{array}{l}\text { Oे } \\
\stackrel{+}{0} \\
\infty \\
\stackrel{0}{+} \\
\stackrel{i}{-1}\end{array}$ & 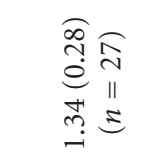 & 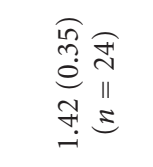 & 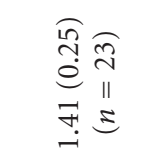 \\
\hline 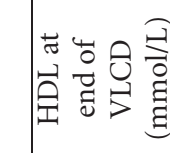 & | & | & I & | & 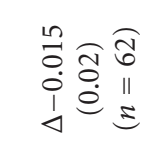 & 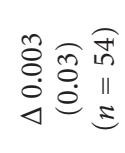 & 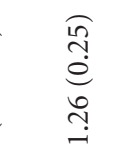 & 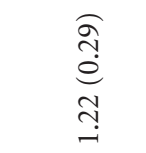 & 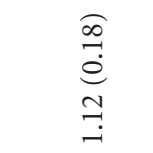 & 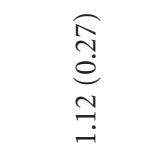 & $\stackrel{e}{a}$ \\
\hline 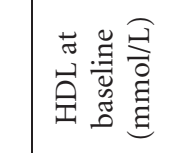 & | & | & 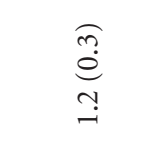 & 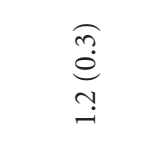 & 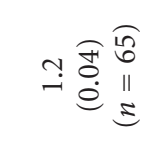 & 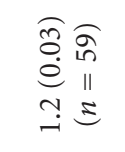 & 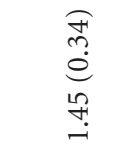 & 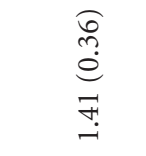 & 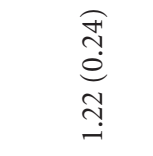 & 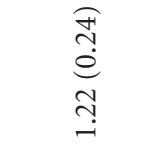 & $\underset{v}{\mathfrak{e}}$ \\
\hline 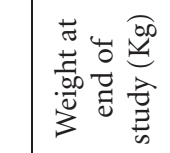 & 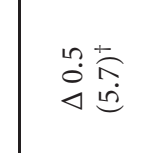 & 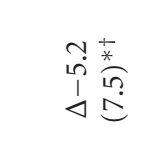 & $\begin{array}{l}\overbrace{\Xi}^{*} \\
\vdots \\
\vdots \\
\alpha\end{array}$ & 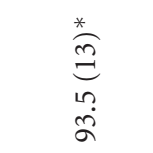 & 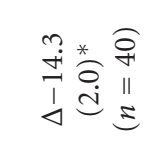 & 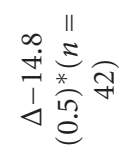 & 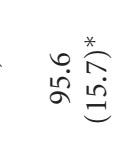 & $\vec{\sigma} \stackrel{*}{\stackrel{*}{n}}$ & 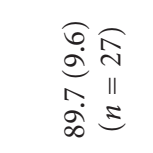 & 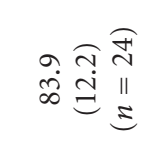 & 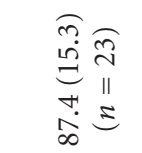 \\
\hline 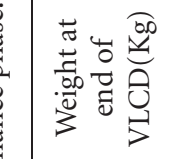 & $\stackrel{*}{\stackrel{*}{ล}} \stackrel{2}{\varrho}$ & 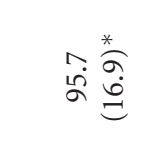 & $\stackrel{\stackrel{\sim}{\exists}}{\exists}$ & 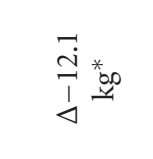 & 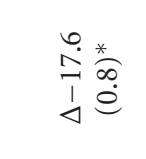 & 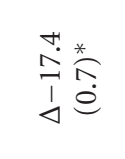 & $\stackrel{\sim}{i} \stackrel{*}{\sigma}$ & 棺 & $\begin{array}{l}\overbrace{1}^{*} \\
\stackrel{0}{0} \\
0 \\
\dot{0} \\
\infty\end{array}$ & \begin{tabular}{l}
$*$ \\
\multirow{\infty}{*}{} \\
$\infty$ \\
0 \\
0 \\
$\infty$ \\
$\infty$
\end{tabular} & 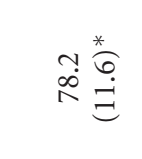 \\
\hline 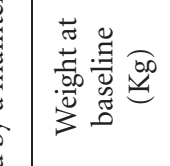 & $\begin{array}{l}\overrightarrow{\text { an }} \\
\text { 官 }\end{array}$ & 봉 & 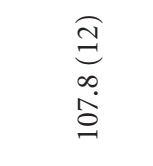 & 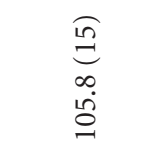 & 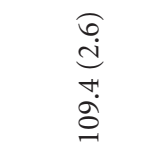 & 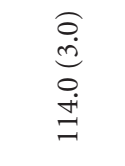 & 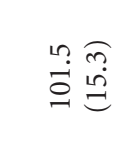 & 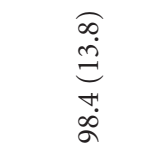 & $\begin{array}{l}\widehat{\infty} \\
\stackrel{a}{ } \\
\circ \\
\stackrel{i}{\sigma}\end{array}$ & $\begin{array}{l}\text { ọ } \\
\stackrel{0}{0} \\
\stackrel{i}{a} \\
\text { à }\end{array}$ & $\begin{array}{l}\infty \\
\stackrel{\infty}{0} \\
0\end{array}$ \\
\hline 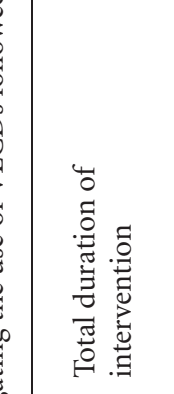 & 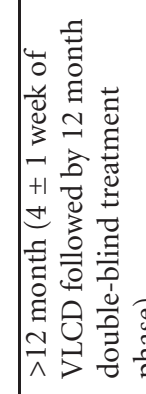 & 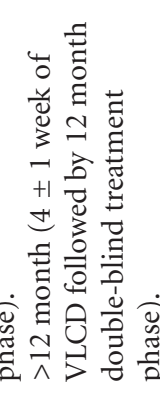 & 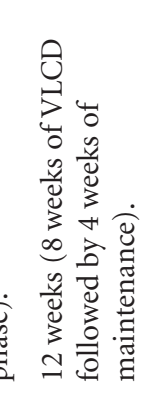 & 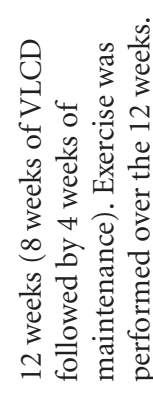 & 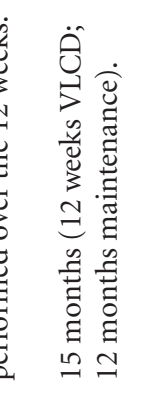 & 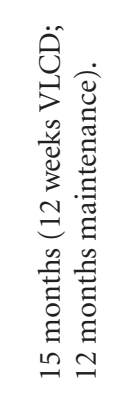 & 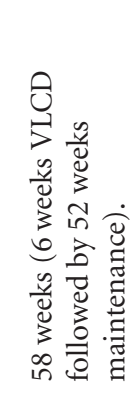 & 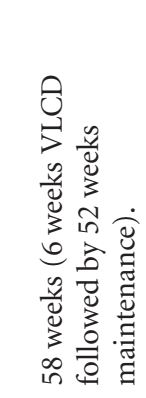 & 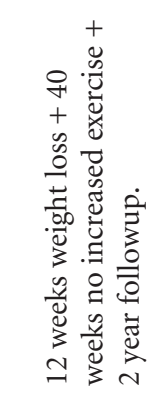 & 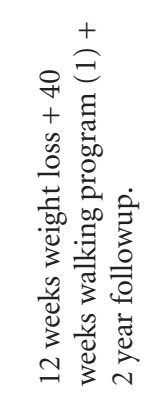 & 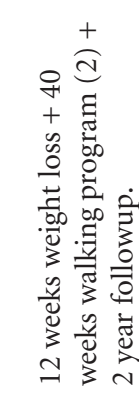 \\
\hline 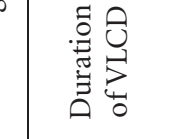 & 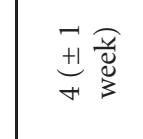 & 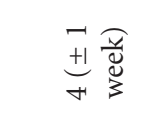 & $\begin{array}{l}\text { च̆ } \\
\vdots \\
\infty\end{array}$ & $\begin{array}{l}\text { चू } \\
\sum_{\infty}^{\circ} \\
\infty\end{array}$ & 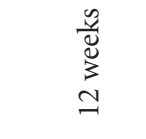 & 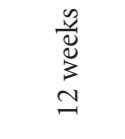 & $\begin{array}{l}\frac{\tilde{g}}{\tilde{u}} \\
\frac{3}{6}\end{array}$ & $\begin{array}{l}\frac{\tilde{y}}{\tilde{u}} \\
\tilde{3} \\
6\end{array}$ & $\begin{array}{l}\frac{n}{\tilde{u}} \\
\vdots \\
\infty \\
\infty\end{array}$ & $\begin{array}{l}\frac{\infty}{\tilde{u}} \\
\bigsqcup_{3}^{0} \\
\infty\end{array}$ & $\frac{\ddot{U}}{\tilde{\Xi}}$ \\
\hline 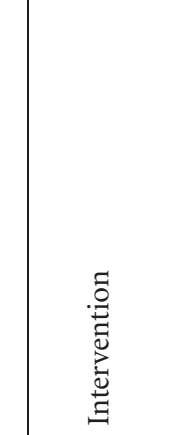 & 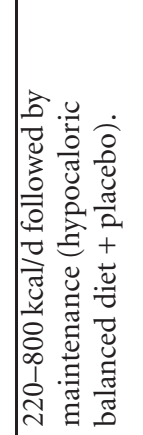 & 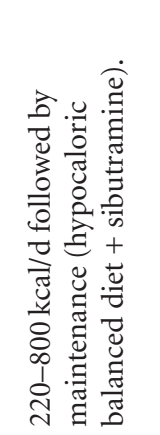 & 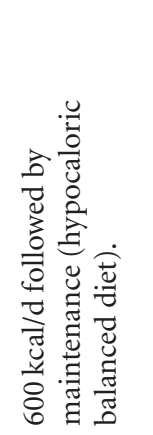 & 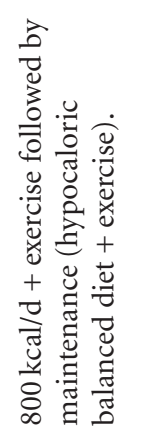 & 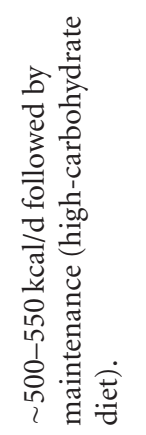 & 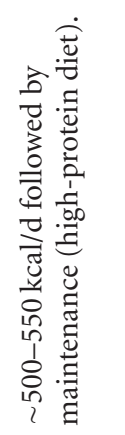 & 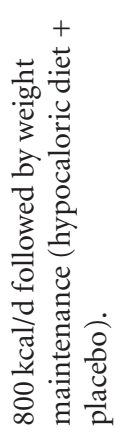 & 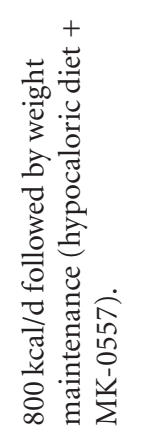 & 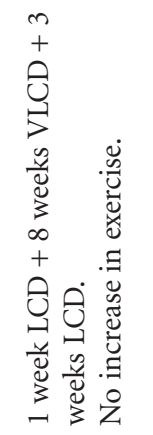 & 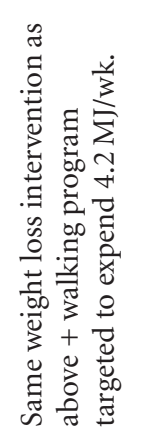 & 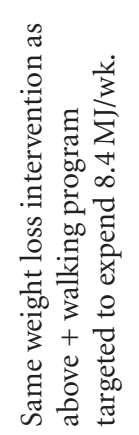 \\
\hline 竞营 & $\stackrel{\infty}{\sim}$ & 으 & $\stackrel{2}{2}$ & 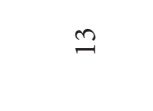 & $\stackrel{n}{m}$ & $\stackrel{n}{m}$ & $\tilde{m}$ & $\hat{m}$ & 0 & 0 & \\
\hline$z$ & $\stackrel{\infty}{\wedge}$ & $\tilde{\infty}$ & ণे & $\stackrel{\sim}{\sim}$ & $R$ & $\nabla$ & $\stackrel{゚}{\beth}$ & $\stackrel{\infty}{\sim}$ & $\stackrel{\infty}{\sim}$ & $\stackrel{\sim}{\sim}$ & \\
\hline D & 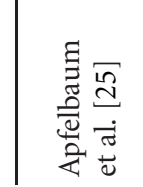 & 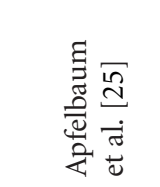 & 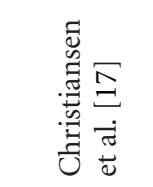 & 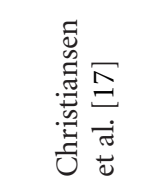 & 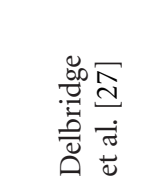 & 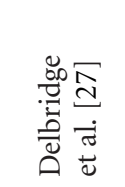 & 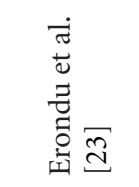 & 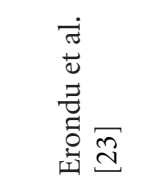 & 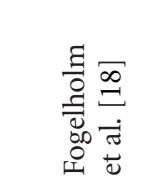 & 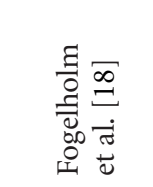 & 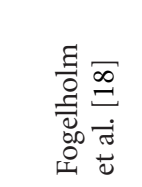 \\
\hline
\end{tabular}




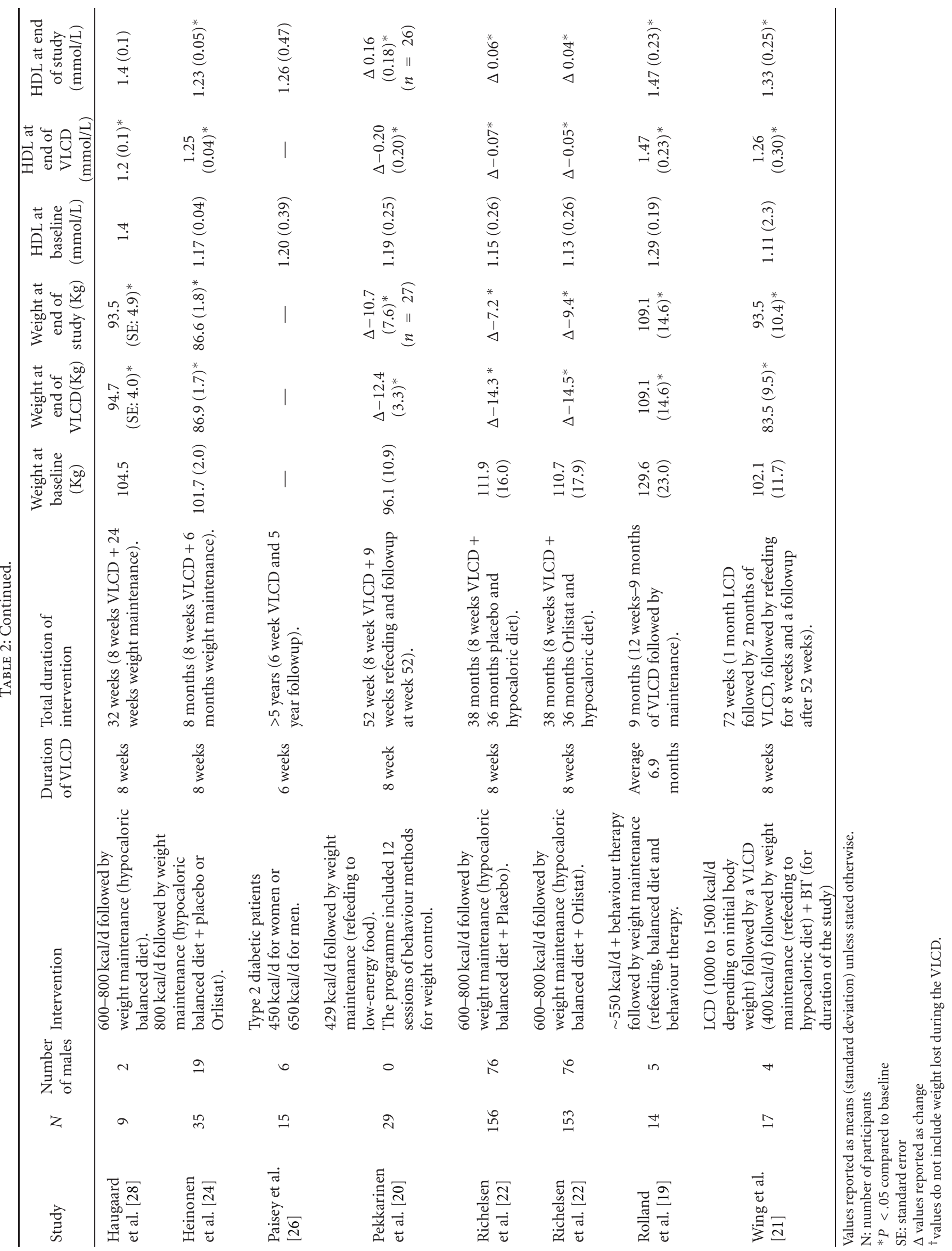




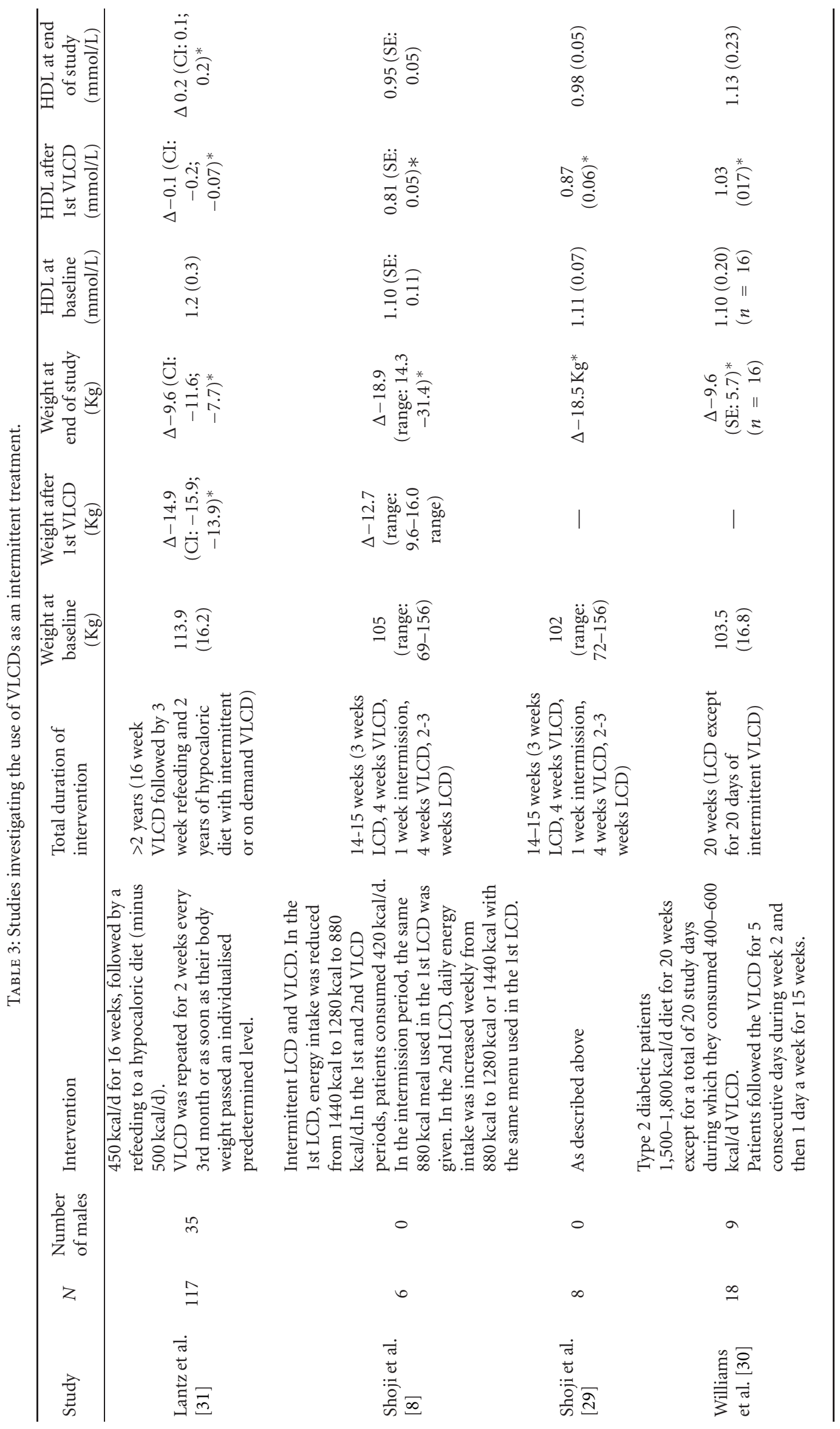




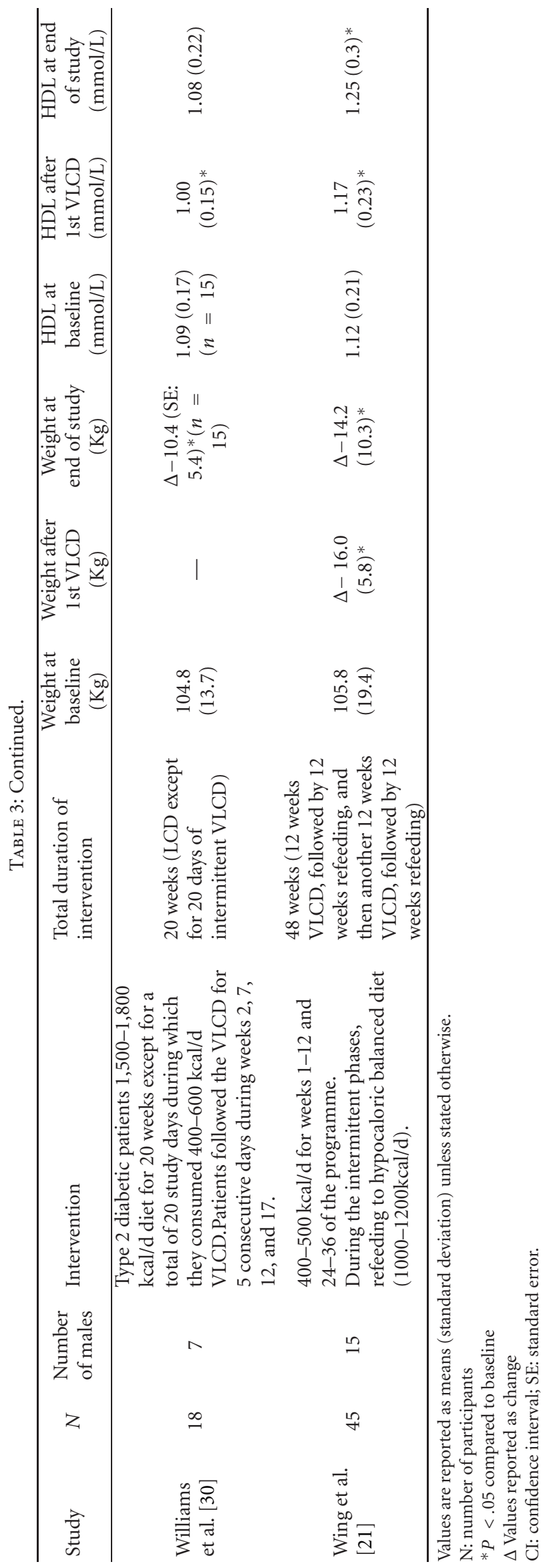


combination of liquid diet supplemented with lean meats or low-starch vegetable.

From the studies presented in this paper, it appears that HDL initially decreases during weight loss treatment using VLCDs, but then either increases back to baseline levels or result in an overall improvement of HDL levels during weight maintenance. This biphasic response may explain the discrepancies between the studies presented here. HDL particles are comprised of several subfractions: $\mathrm{HDL}_{2}, \mathrm{HDL}_{3}$, apolipoprotein A-I (Apo A-I), apolipoprotein A-II (Apo AII), and pre- $\beta_{1}$. HDL 2 , Apo A-I, and pre- $\beta_{1}$ are associated with cardioprotection and are found to be reduced in the obesity [35]. In response to the VLCD, Shoji et al. [29] observed that the initial decrease in $\mathrm{HDL}_{2}$ was associated with decreased lecithin cholesterol ester transfer protein (LCAT) (which plays an important role in the formation of $\mathrm{HDL}_{2}$ [36]) while Apo A-1 increased. Apo A-1 activates LCAT, which may explain the delayed improvement in HDL [29]. This observation is consistent with the meta-analysis by Dattilo and Kris-Etherton [37] that reported a decrease during active weight loss but an increase in HDL when weight loss was stabilised after a dietary intervention. More information, however, will be required to correlate weight, Apo-A1, and LCAT metabolism changes with HDL changes to allow a definitive comment on this parameter.

It has been suggested that exercise increases HDL cholesterol. In the studies reviewed here, Christiansen et al. displayed a significant increase in HDL. Fogelholm et al. [18] showed a similar trend, but this was not significant at 2 years. This was probably due to the fact that there was no difference in the number of steps taken between the different interventions at the end of the study, suggesting a poor compliance to exercise. Exercise has been associated with increases in HDL cholesterol, although a relatively highintensity exercise is required for significant changes in HDL. It is thought that the increase in HDL in response to exercise is due to an increase in the activity of lipoprotein lipase (which transfers lipids to HDL) and a reduction in the activity of hepatic lipase (which removes lipids HDL in the blood) [38]. It has also been suggested that the degree of change in HDL in response to exercise may be dependent on genetic factors $[39,40]$.

The main concern about the use of VLCDs is the weight regain that is often observed after use. To date, there has not been any solution to the almost inevitable long-term weight regain associated with weight loss in general. While also focusing on the prevention of weight regain, however, it is important to also concentrate on maintaining the health benefits (i.e., increased HDL) associated with the initial weight loss. The study by Delbridge et al. [27] was quite interesting where they compared a high-carbohydrate to a high-protein weight-maintenance diet for a period of 12 months. Although there was a trend for an increase in HDL in both groups, there was no difference between the two weight-maintenance diets. This is inconsistent with the literature where it has been shown that at 12 months, HDL levels are generally higher in patients who followed a high-protein diet compared to a high-carbohydrate diet [41]. The effect of the weight maintenance diet in the study by Delbridge et al. [27] may have been blurred by the initial weight loss achieved using the VLCD, or by lack of compliance to the diet.

The effect of medication for weight maintenance on HDL could also be interesting to investigate. Four studies incorporated the use of obesity medication. One of the studies investigating the use of Orlistat showed no significant difference in HDL levels in the longer term [22] while the other study clumped their data for the placebo and Orlistat group for their overall analysis [24]. Erondu et al. [23] did not report a significant change in HDL at 1year followup after randomisation to the neuropeptide-Y receptor agonist MK-0557. The study investigating the use of Sibutramine for weight maintenance, however, did report a long-term improvement in HDL. From other studies, it would appear that Sibutramine $[25,42]$ and dexfenfluramine [43] may be more effective for weight maintenance after a VLCD compared to Orlistat $[15,22,44]$ or MK-0557 [23]. More research into the effect of Sibutramine on weight maintenance and the benefits on HDL should be pursued.

The main difficulty encountered was the heterogeneity of the studies reviewed here. There was a wide variety of intervention strategies, duration, and baseline HDL levels (ranging from 1.05 to $1.50 \mathrm{mmol} / \mathrm{L}$ ) which makes it very difficult to reach clear conclusions and supply recommendations for best practice. In addition, there is a paucity of evidence for populations at increased risk of coronary artery disease such as type 2 diabetics, gender, and different ethnic groups. There is an obvious need for more research and better defined intervention strategies for the use of VLCDs in long-term treatment.

\section{Conclusion}

The results from this paper suggest that HDL can be improved in the longer term by using a combination of a VLCD followed by a weight-maintenance period using a hypocaloric diet. Nevertheless, the studies reviewed here are very heterogeneous and more research needs to be carried out to define the short-term effect of weight loss on HDL levels as well as investigating the effects of VLCDs in groups at higher risk of coronary heart disease such as type 2 diabetics.

\section{Conflict of Interests}

Some of the authors research has been funded by LighterLife UK Limited and Professor I. Broom is the medical director for this company.

\section{References}

[1] T. B. van Itallie, "Liquid protein mayhem," Journal of the American Medical Association, vol. 240, no. 2, pp. 144-145, 1978.

[2] Center for Disease Control, Liquid Protein Diets. Public Health Service Report, Center for Disease Control, Atlanta, Ga, USA, 1979. 
[3] W. H. Saris, "Very-low-calorie diets and sustained weight loss," Obesity Research, vol. 9, supplement 4, pp. 295S-301S, 2001.

[4] T. A. Wadden, "Treatment of obesity by moderate and severe caloric restriction: results of clinical research trials," Annals of Internal Medicine, vol. 119, no. 7, part 2, pp. 688-693, 1993.

[5] A. Avenell, J. Broom, T. J. Brown et al., "Systematic review of the long-term effects and economic consequences of treatments for obesity and implications for health improvement," Health Technology Assessment, vol. 8, no. 21, pp. 1-182, 2004.

[6] A. D. Mooradian, M. J. Haas, K. R. Wehmeier, and N. C. W. Wong, "Obesity-related changes in high-density lipoprotein metabolism," Obesity, vol. 16, no. 6, pp. 1152-1160, 2008.

[7] B. S. Fong, P. O. Rodrigues, and A. M. Salter, "Characterization of high density lipoprotein binding to human adipocyte plasma membranes," The Journal of Clinical Investigation, vol. 75, no. 6, pp. 1804-1812, 1985.

[8] T. Shoji, Y. Nishizawa, H. Koyama et al., "Lipoprotein metabolism in normolipidemic obese women during very low calorie diet: changes in high density lipoprotein," Journal of Nutritional Science and Vitaminology, vol. 37, pp. S57-64, 1991.

[9] P. P. Toth, "High-density lipoprotein as a therapeutic target: clinical evidence and treatment strategies," American Journal of Cardiology, vol. 96, no. 9, pp. 50K-58K, 2005.

[10] A. R. Tall, "Plasma high density lipoproteins. Metabolism and relationship to atherogenesis," The Journal of Clinical Investigation, vol. 86, no. 2, pp. 379-384, 1990.

[11] A. D. Mooradian, M. J. Haas, and N. C. W. Wong, "The effect of select nutrients on serum high-density lipoprotein cholesterol and apolipoprotein A-I levels," Endocrine Reviews, vol. 27, no. 1, pp. 2-16, 2006.

[12] K. Hong, Z. Li, H. J. Wang, R. Elashoff, and D. Heber, "Analysis of weight loss outcomes using VLCD in black and white overweight and obese women with and without metabolic syndrome," International Journal of Obesity, vol. 29, no. 4, pp. 436-442, 2005.

[13] K. Clément, N. Viguerie, C. Poitou et al., "Weight loss regulates inflammation-related genes in white adipose tissue of obese subjects," The FASEB Journal, vol. 18, no. 14, pp. 1657-1669, 2004.

[14] S. B. Haugaard, A. Vaag, C.-E. Høy, and S. Madsbad, "Desaturation of skeletal muscle structural and depot lipids in obese individuals during a very-low-calorie diet intervention," Obesity, vol. 15, no. 1, pp. 117-125, 2007.

[15] D. E. Laaksonen, S. Kainulainen, A. Rissanen, and L. Niskanen, "Relationships between changes in abdominal fat distribution and insulin sensitivity during a very low calorie diet in abdominally obese men and women," Nutrition, Metabolism and Cardiovascular Diseases, vol. 13, no. 6, pp. 349-356, 2003.

[16] W.-Y. Lin, C.-H. Wu, N.-F. Chu, and C.-J. Chang, "Efficacy and safety of very-low-calorie diet in Taiwanese: a multicenter randomized, controlled trial," Nutrition, vol. 25, no. 11-12, pp. 1129-1136, 2009.

[17] T. Christiansen, S. K. Paulsen, J. M. Bruun, S. B. Pedersen, and B. Richelsen, "Exercise training versus diet-induced weightloss on metabolic risk factors and inflammatory markers in obese subjects: a 12-week randomized intervention study," American Journal of Physiology, vol. 298, no. 4, pp. E824-E831, 2010.

[18] M. Fogelholm, K. Kukkonen-Harjula, A. Nenonen, and M. Pasanen, "Effects of walking training on weight maintenance after a very-low- energy diet in premenopausal obese women: a randomized controlled trial," Archives of Internal Medicine, vol. 160, no. 14, pp. 2177-2184, 2000.
[19] C. Rolland, M. Hession, S. Murray, A. Wise, and I. Broom, "Randomized clinical trial of standard dietary treatment versus low-carbohydrate/high-protein diet or the LighterLife Programme in the management of obesity," Journal of Diabetes, vol. 1, 217 pages, 2009.

[20] T. Pekkarinen, I. Takala, and P. Mustajoki, "Weight loss with very-low-calorie diet and cardiovascular risk factors in moderately obese women: one-year follow-up study including ambulatory blood pressure monitoring," International Journal of Obesity, vol. 22, no. 7, pp. 661-666, 1998.

[21] R. R. Wing, M. D. Marcus, R. Salata, L. H. Epstein, S. Miaskiewicz, and E. H. Blair, "Effects of a very-low-calorie diet on long-term glycemic control in obese type 2 diabetic subjects," Archives of Internal Medicine, vol. 151, no. 7, pp. 1334-1340, 1991.

[22] B. Richelsen, S. Tonstad, S. Rössner et al., "Effect of orlistat on weight regain and cardiovascular risk factors following a very-low-energy diet in abdominally obese patients: a 3-year randomized, placebo-controlled study," Diabetes Care, vol. 30, no. 1, pp. 27-32, 2007.

[23] N. Erondu, T. Wadden, I. Gantz et al., "Effect of NPY5R antagonist MK-0557 on weight regain after very-low-calorie diet-induced weight loss," Obesity, vol. 15, no. 4, pp. 895-905, 2007.

[24] M. V. Heinonen, D. E. Laaksonen, T. Karhu et al., "Effect of diet-induced weight loss on plasma apelin and cytokine levels in individuals with the metabolic syndrome," Nutrition, Metabolism and Cardiovascular Diseases, vol. 19, no. 9, pp. 626-633, 2009.

[25] M. Apfelbaum, P. Vague, O. Ziegler, C. Hanotin, F. Thomas, and E. Leutenegger, "Long-term maintenance of weight loss after a very-low-calorie diet: a randomized blinded trial of the efficacy and tolerability of sibutramine," The American Journal of Medicine, vol. 106, no. 2, pp. 179-184, 1999.

[26] R. B. Paisey, J. Frost, P. Harvey et al., "Five year results of a prospective very low calorie diet or conventional weight loss programme in type 2 diabetes," Journal of Human Nutrition and Dietetics, vol. 15, no. 2, pp. 121-127, 2002.

[27] E. A. Delbridge, L. A. Prendergast, J. E. Pritchard, and J. Proietto, "One-year weight maintenance after significant weight loss in healthy overweight and obese subjects: does diet composition matter?" The American Journal of Clinical Nutrition, vol. 90, no. 5, pp. 1203-1214, 2009.

[28] S. B. Haugaard, A. Vaag, H. Mu, and S. Madsbad, "Skeletal muscle structural lipids improve during weight-maintenance after a very low calorie dietary intervention," Lipids in Health and Disease, vol. 8, article 34, 2009.

[29] T. Shoji, Y. Nishizawa, H. Koyama et al., "High-densitylipoprotein metabolism during a very-low-calorie diet," The American Journal of Clinical Nutrition, vol. 56, no. 1, pp. 297S298S, 1992.

[30] K. V. Williams, M. L. Mullen, D. E. Kelley, and R. R. Wing, "The effect of short periods of caloric restriction on weight loss and glycemic control in type 2 diabetes," Diabetes Care, vol. 21, no. 1, pp. 2-8, 1998.

[31] H. Lantz, M. Peltonen, L. Ågren, and J. S. Torgerson, "Intermittent versus on-demand use of a very low calorie diet: a randomized 2-year clinical trial," Journal of Internal Medicine, vol. 253, no. 4, pp. 463-471, 2003.

[32] G. D. Foster, T. A. Wadden, F. J. Peterson, K. A. Letizia, S. J. Bartlett, and A. M. Conill, "A controlled comparison of three very-low-calorie diets: effects on weight, body composition, and symptoms," The American Journal of Clinical Nutrition, vol. 55, no. 4, pp. 811-817, 1992. 
[33] M. Ohno, J. Miura, K. Arai, S. Tsukahara, and Y. Ikeda, "The efficacy and metabolic effects of two different regimens of very low calorie diet," International Journal of Obesity, vol. 13, no. 2, pp. 79-85, 1989.

[34] S. Rössner and H. Flaten, "VLCD versus LCD in long-term treatment of obesity," International Journal of Obesity, vol. 21, no. 1, pp. 22-26, 1997.

[35] S. Rashid and J. Genest, "Effect of obesity on high-density lipoprotein metabolism," Obesity, vol. 15, no. 12, pp. 28752888, 2007.

[36] M. Dobiášová and J. Frohlich, “Understanding the mechanism of LCAT reaction may help to explain the high predictive value of LDL/HDL cholesterol ratio," Physiological Research, vol. 47, no. 6, pp. 387-397, 1998.

[37] A. M. Dattilo and P. M. Kris-Etherton, "Effects of weight reduction on blood lipids and lipoproteins: a meta-analysis," The American Journal of Clinical Nutrition, vol. 56, no. 2, pp. 320-328, 1992.

[38] O. L. Svendsen, C. Hassager, and C. Christiansen, "Six months' follow-up on exercise added to a short-term diet in overweight postmenopausal women-effects on body composition, resting metabolic rate, cardiovascular risk factors and bone," International Journal of Obesity, vol. 18, no. 10, pp. 692-698, 1994.

[39] K. R. Wilund, R. E. Ferrell, D. A. Phares, A. P. Goldberg, and J. M. Hagberg, "Changes in high-density lipoproteincholesterol subfractions with exercise training may be dependent on cholesteryl ester transfer protein (CETP) genotype," Metabolism: Clinical and Experimental, vol. 51, no. 6, pp. 774778, 2002.

[40] A. Halverstadt, D. A. Phares, R. E. Ferrell, K. R. Wilund, A. P. Goldberg, and J. M. Hagberg, "High-density lipoproteincholesterol, its subfractions, and responses to exercise training are dependent on endothelial lipase genotype," Metabolism: Clinical and Experimental, vol. 52, no. 11, pp. 1505-1511, 2003.

[41] M. Hession, C. Rolland, U. Kulkarni, A. Wise, and J. Broom, "Systematic review of randomized controlled trials of lowcarbohydrate vs. low-fat/low-calorie diets in the management of obesity and its comorbidities," Obesity Reviews, vol. 10, no. 1, pp. 36-50, 2009.

[42] E. M.H. Mathus-Vliegen, "Long-term maintenance of weight loss with sibutramine in a GP setting following a specialist guided very-low-calorie diet: a double-blind, placebocontrolled, parallel group study," European Journal of Clinical Nutrition, vol. 59, supplement 1, pp. S31-S39, 2005.

[43] N. Finer, S. Finer, and R. P. Naoumova, "Drug therapy after very-low-calorie diets," The American Journal of Clinical Nutrition, vol. 56, no. 1, pp. 195S-198S, 1992.

[44] J. D. LeCheminant, D. J. Jacobsen, M. A. Hall, and J. E. Donnelly, "A comparison of meal replacements and medication in weight maintenance after weight loss," Journal of the American College of Nutrition, vol. 24, no. 5, pp. 347-353, 2005. 


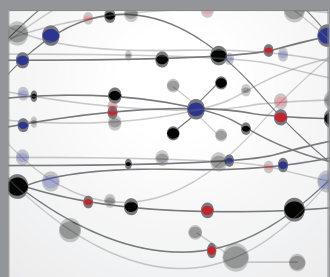

The Scientific World Journal
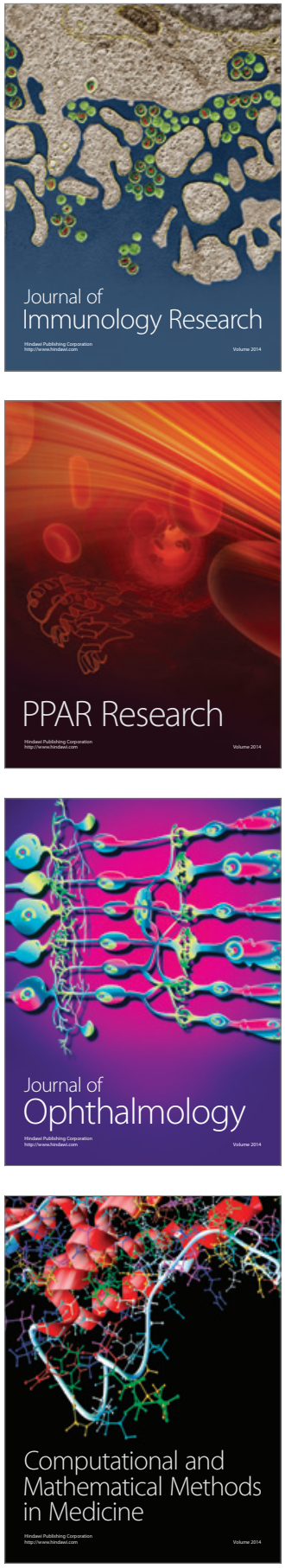

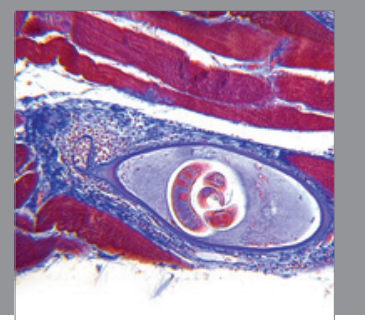

Gastroenterology

Research and Practice
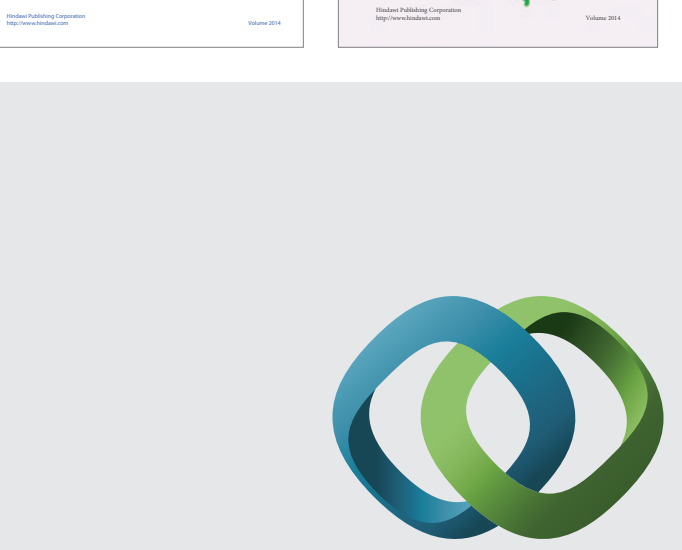

\section{Hindawi}

Submit your manuscripts at

http://www.hindawi.com
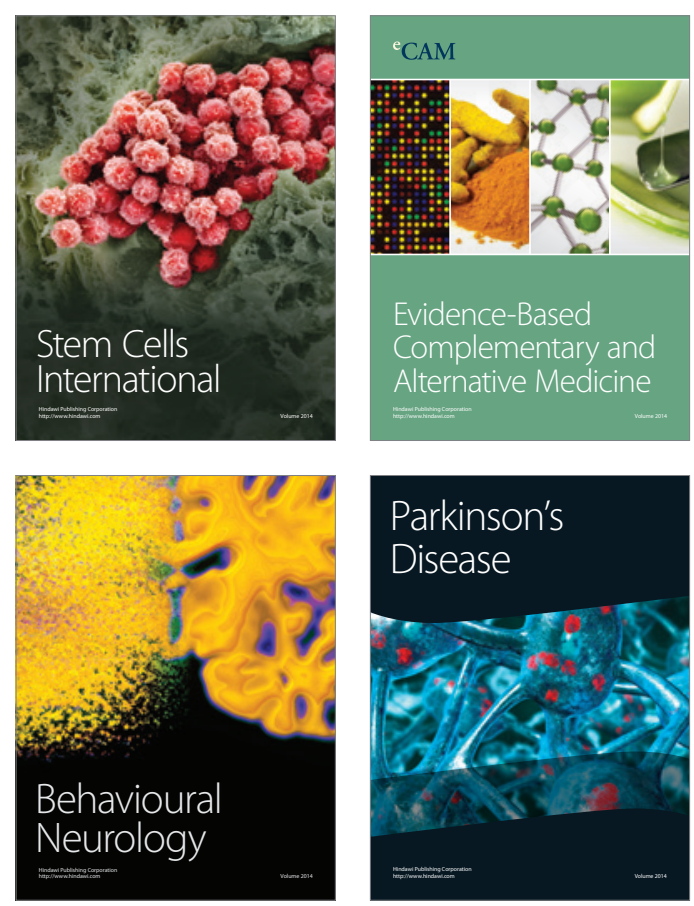

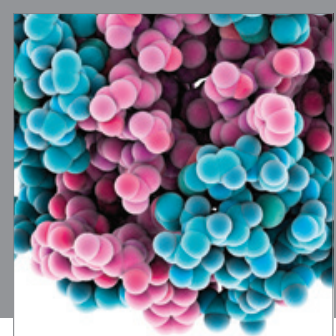

Journal of
Diabetes Research

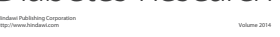

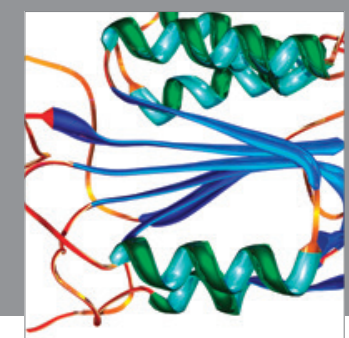

Disease Markers
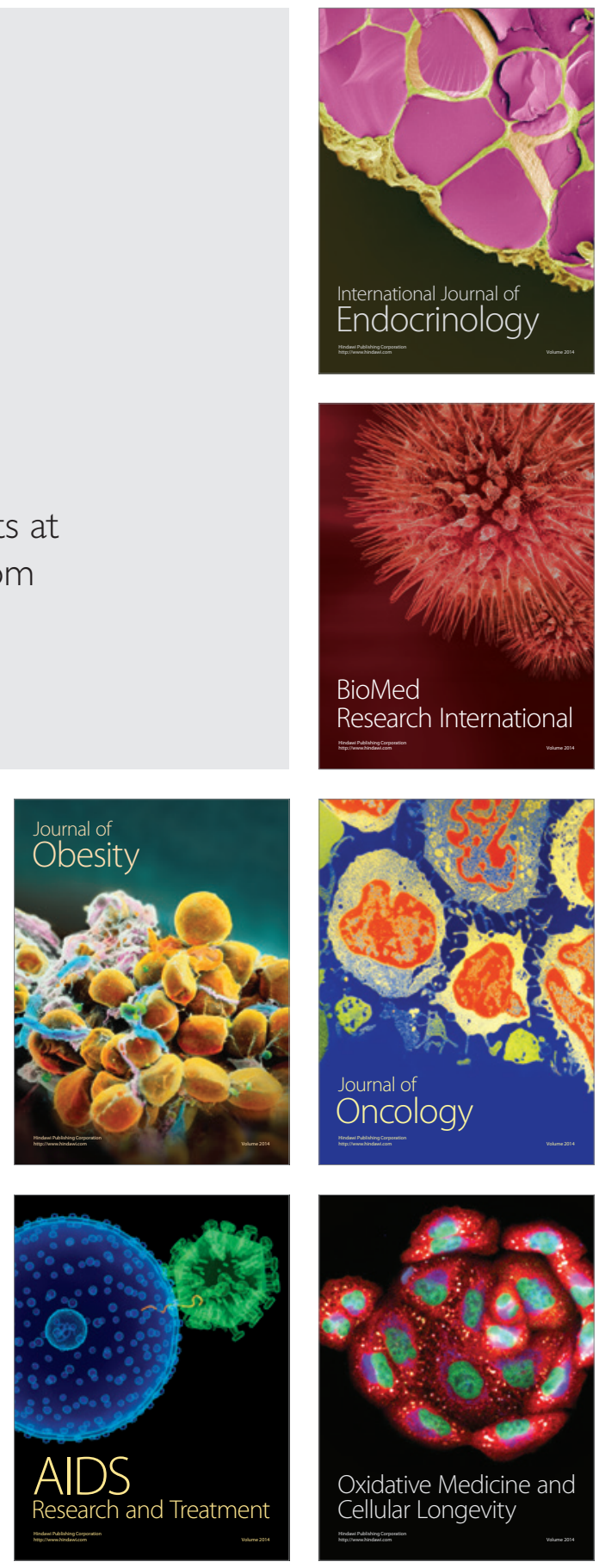